\title{
Síndrome de ovarios poliquísticos: abordaje diagnóstico y terapéutico.
}

\section{Revisión}

Miguel A. Vargas-Carrillo ${ }^{1,2}$, Gabriel Sánchez-Buenfil ${ }^{3}$, Jorge Herrera-Polanco ${ }^{1}$, Lizardo Vargas-Ancona ${ }^{1}$

${ }^{1}$ División de Medicina Interna, ${ }^{2}$ Servicio de Endocrinología, ${ }^{3}$ División de Gineco-Obstetricia, Hospital General "Agustín O'Horán", Secretaria de Salud, Mérida, Yucatán, México.

\section{RESUMEN.}

El síndrome de ovarios poliquísticos es una entidad frecuente en la mujer joven. La importancia de su diagnóstico radica en la exclusión de patologías potencialmente graves, como las neoplasias ováricas y adrenales o la hiperplasia adrenal congénita y en el reto que representa para los clínicos, en cuanto a prevención se refiere, por su nexo fisiopatológico con los estados de resistencia a la insulina e hiperinsulinemia compensatoria. Hoy sabemos que estas mujeres tienen riesgos significativamente elevados de diabetes tipo 2, hipertensión arterial y cardiopatía isquémica, lo que repercute en un aumento en su mortalidad. La presente revisión ofrece una actualización del tema, haciendo énfasis en los aspectos clínicos y las implicaciones de su fisiopatología en las decisiones médicas.

(Rev Biomed 2003; 14:191-203)

Palabras clave: hiperandrogenismo, hirsutismo, ovario, hiperinsulinemia.

\section{SUMMARY.}

The polycystic ovary syndrome: Diagnostic and therapeutic approach.

The polycystic ovary syndrome is a very frequent entity in young women. The importance of its diagnosis radicates in it's exclusion with potentially serious pathologies such as ovarian and adrenal gland tumors or with congenital adrenal hiperplasia, and in the challenge that it represents to phycisians, when it comes to prevention because of its physiopathological link with the insulin resistant state and compensatory hiperinsulinemia. Today we know that these women have significantly higher risks of presenting diabetes type 2, arterial hipertension and isquemic heart disease which increases their mortality rates. This next review offers an up-date on the subject emphasising the clinical aspects and the implications of physiopathology in medical decisions.

\section{(Rev Biomed 2003; 14:191-203)}

Key words: hiperandrogenism, hirsutism, ovary, hiperinsulinemia.

\section{INTRODUCCIÓN.}

El "hiperandrogenismo" representa la primera causa de consulta endocrinológica en mujeres que cursan la edad reproductiva e incluye en su contexto clínico la presencia de obesidad, irregularidades menstruales, anovulación crónica, acné, hirsutismo, seborrea, acanthosis nigricans y alopecia, entre otras manifestaciones. La mayoría de estos casos se

Solicitud de sobretiros: Dr. Miguel A. Vargas-Carrillo, Departamento de Enseñanza y Capacitación, Hospital General "Agustín O'Horan", Calle 59-A x Av. Itzaez, C.P. 97000, Mérida, Yucatán, México. E-mail: dr_mvargas@hotmail.com 
MA Vargas-Carrillo, G Sánchez-Buenfil, J Herrera-Polanco, L Vargas-Ancona.

relacionan con un trastorno de la función ovárica conocido como síndrome de ovarios poliquísticos (SOP) (1).

La importancia del SOP en nuestra práctica médica cotidiana podemos analizarla desde diferentes puntos de vista. Por una parte, clínicamente involucra a médicos con diferentes especialidades y la razón de esto es su amplio espectro clínico; hablamos de médicos generales, dermatólogos, ginecólogos, internistas, nutriólogos y endocrinólogos. El otro punto relevante lo constituye el hecho de que estas mujeres tienen riesgos significativamente incrementados de diabetes mellitus tipo 2 (DM tipo 2), hipertensión arterial (HTA), dislipidemia y cardiopatía isquémica, lo cual incide en una mayor mortalidad (2-4). Esta asociación epidemiológica tiene su base en la resistencia a la insulina y la hiperinsulinemia compensatoria en el hoy conocido como síndrome metabólico que incluye entre sus componentes a la hiperestimulación ovárica (5).

El motivo de la presente revisión es proporcionar una actualización de las implicaciones de la fisiopatología del SOP en los riesgos de salud y la toma de decisiones en el consultorio.

\section{EPIDEMIOLOGIA.}

El SOP tiene una prevalencia del 5-10\% en la población general (6). El 50-65\% de las pacientes con SOP son obesas y se considera que el 35-45\% de ellas presentará intolerancia a la glucosa durante una curva de tolerancia a la glucosa, con un riesgo de desarrollar DM tipo 2 del doble a lo esperado para su edad (7,8). A través de estudios con pinzamiento euglucémico se considera que la sensibilidad a la insulina está reducida en un 35-40\% en este grupo de pacientes, lo que es similar a lo referido para pacientes con DM tipo 2 (9).

El papel de la excesiva producción de andrógenos en el desarrollo de la enfermedad cardiovascular ha sido demostrado epidemiológicamente: Sabemos que estas mujeres son con mayor frecuencia diabéticas, hipertensas, obesas y dislipidémicas $(10,11)$. Retrospectivamente se ha reportado un aumento en el riesgo de presentar HTA de hasta cuatro veces en las pacientes con SOP (9), así como un aumento de 7.4 veces en el riesgo relativo de sufrir infarto agudo al miocardio (IAM), en ambos casos apareados con controles (12). En un estudio de 143 pacientes menores de 60 años que requirieron de angiografía coronaria, se encontró que las mujeres con evidencia clínica de SOP tenían el doble de segmentos involucrados de las arterias coronarias con una estenosis mayor del 50\% (13). De acuerdo a los datos del Nurse Health Study la irregularidad menstrual incrementó en un 50\% el riesgo de sufrir IAM (14).

Aparte de las anormalidades cuantitativas en el perfil de lípidos existen cambios cualitativos no detectados en los estudios de laboratorio en nuestra rutina médica y que parecen hacer al colesterol de baja densidad (C-LDL) de estos pacientes más aterogénico (15). En presencia de resistencia a la insulina la hidrólisis de los triglicéridos es incompleta, lo que hace "más pequeñas" a las moléculas de colesterol LDL (16). Estas partículas se oxidan con mayor facilidad lo que favorece su incorporación a las paredes arteriales en donde son reconocidas por macrófagos y transportadas al espacio subendotelial (17). Allí tienen propiedades citotóxicas por lo que provocan daño en forma directa, lo que favorece la adhesión y agregación plaquetaria, que a su vez provoca la proliferación de las células de músculo liso por la liberación a nivel local de factores de crecimiento. Todo esto hace progresar la placa fibrosa, a la vez que la hace vulnerable (18). La ruptura de estas placas es responsable de la mayoría de los eventos trombóticos en pacientes con enfermedad aterosclerosa (19-21).

Es de llamar la atención el hecho de que desde la juventud estas pacientes muestran disminución de la sensibilidad a la insulina e hiperinsulinemia (22), elevación del activador del plasminógeno tisular (tPA), lo que denota una alteración de la fibrinólisis y aumento en los niveles de homocisteína, ambos cambios relacionados con un aumento en el riesgo de cardiopatía isquémica $(23,24)$.

Quienes consiguen un embarazo presentan mayores riesgos de preclampsia (32\% vs $3.7 \%$ en normales (25) y de Diabetes Gestacional (31\% vs 3\%) (26).

\section{Revista Biomédica}




\section{Síndrome de ovarios poliquísticos.}

No contamos con trabajos regionales que sugieran nuestra prevalencia de SOP. Sin embargo, al comparar los resultados de los estudios epidemiológicos realizados por el grupo de FUNSALUD en nuestro estado y los obtenidos por la encuesta nacional de enfermedades crónicas, observamos en la población urbana de Yucatán, México una mayor presencia de las patologías habitualmente relacionadas con el SOP. Las cifras de obesidad en mujeres se encuentran muy por arriba del promedio nacional (41.4\% vs $21.4 \%$ ) (27). Lo mismo ocurre en relación con las cifras de DM tipo 2 $(14.8 \%$ vs $8.2 \%)(28,29)$ y dislipidemia: colesterol total $\geq 200 \mathrm{mg} / \mathrm{dL} 41.2 \%$ vs $29.3 \%$ (30).

Esto sugiere que la prevalencia de SOP en Yucatán pudiera ser mayor que la referida en otros sitios. Los factores determinantes en este sentido incluirían el racial y el ambiental.

\section{FISIOPATOLOGIA.}

Las dos principales anormalidades hormonales halladas en los pacientes con SOP incluyen la elevación de los niveles circulantes de hormona luteinizante $(\mathrm{LH})$ e insulina (31). La hipótesis más aceptable sugiere un sinergismo entre ambas, lo cual termina por provocar en el ovario, atresia folicular e incapacidad para la formación de estrógenos. En este contexto, la hiperestimulación ovárica por la insulina, provocaría hiperandrogenismo (32). Intentaremos detallar en las siguientes líneas algunos aspectos importantes que han llevado a esta afirmación.

Se sabe ahora, que aparte de la FSH y la LH secretadas por la hipófisis, la insulina y algunas otras hormonas están involucradas en la regulación de la función ovárica $(33,34)$. Asimismo, se han identificado en el ovario humano receptores para la insulina y los factores de crecimiento similares a la insulina tipo 1 (IGF-1), los cuales son homólogos de los receptores de la insulina en un $40 \%$ y, aunque con menor afinidad, se unen a ella en presencia de ciertas patologías que cursan con estados de resistencia a la insulina e hiperinsulinemia compensatoria. A modo de círculo vicioso, la hiperinsulinemia incrementa la formación de receptores IGF-1 (35). La hipófisis también contiene receptores para la insulina y bajo su estímulo se incrementa la secreción de LH.

El hecho paradójico de que en pacientes con resistencia a la insulina, el ovario permanezca con una sensibilidad normal e incluso incrementada a la insulina y manifieste esta hiperestimulación con un aumento en la formación de andrógenos exclusivamente, ha sido motivo de numerosos estudios experimentales. Es importante recalcar que el término de resistencia a la insulina hace referencia a una disminución en su poder biológico en cuanto al control glucémico. No necesariamente su eficiencia se encuentre disminuida en todos los tejidos y en algunos sitios la hiperinsulinemia compensatoria llevaría a una hiperestimulación (36).

Las hormonas sexuales son transportadas en su forma inactiva unidas a proteínas específicas. La hiperinsulinemia inhibe la producción de la proteína transportadora de hormonas sexuales (SHBG), lo cual aumenta la proporción de testosterona libre que a su vez representa la forma activa de la hormona. Adicionalmente, la conversión periférica de andrógenos a estrona inhibe la secreción de FSH llevando a ciclos anovulatorios $(26,37)$.

El eje hipotálamo-hipófisis-adrenal también parece involucrado en el síndrome, ya que aproximadamente en $40-70 \%$ de los casos se encuentran elevados los niveles circulantes de algunos andrógenos suprarrenales con respuestas inapropropiadamente altas durante las pruebas de estimulación con adrenocorticotropina (ACTH). Esto pudiera estar relacionado con los niveles elevados de la enzima ovárica citocromo P450c17alfa, la cual está relacionada con la biosíntesis de andrógenos e incrementa los niveles de 17 alfahidroxiprogesterona. $(38,39)$.

La fisiopatología del SOP es compleja y en muchos sentidos no del todo conocida. La resistencia a la insulina tiene un papel central en más del $50 \%$ de los casos, generalmente asociada a obesidad (7). En el resto de los casos las teorías involucran directamente al hipotálamo, a través de cambios en la frecuencia y magnitud de los picos de la hormona liberadora de gonadotropinas, lo cual aumentaría los niveles 


\section{MA Vargas-Carrillo, G Sánchez-Buenfil, J Herrera-Polanco, L Vargas-Ancona.}

circulantes de LH (40), o al ovario y las suprarrenales bajo mecanismos más oscuros (38). La figura 1 ilustra la participación central de la resistencia a la insulina en la hiperestimulación ovárica y adrenal en pacientes con SOP, así como su relación con los riesgos incrementados de DM tipo 2, dislipidemia y cardiopatía isquémica.

\section{MANIFESTACIONES CLINICAS.}

La mayoría de los datos clínicos que aparecen en pacientes con SOP pueden formar parte de otras patologías no necesariamente relacionadas con alguna disfunción hormonal, de tal manera que la asociación de síntomas y signos y no los hechos aislados deben siempre sugerir el diagnóstico. La severidad o la rápida evolución debe alertar a los clínicos a la realización de un diagnóstico diferencial más extenso.

Acné: Aparece frecuentemente en las adolescentes y generalmente para los 15 años de edad el $50 \%$ de ellas lo presentará. Su permanencia de los 20 años en adelante debe considerarse sospechosa. Es imperativo interrogar acerca de irregularidades menstruales, hirsutismo o cualquier otro dato de hiperandrogenismo. Estas pacientes pudieran presentar niveles anormales en los andrógenos séricos, pero los niveles de éstos no tienen correlación con la severidad del acné (3). En estudios realizados en mujeres con acné se ha encontrado datos de SOP hasta en el $45 \%$ de los casos (41).

Hirsutismo: Se define como el crecimiento excesivo del vello corporal terminal en mujeres, en áreas anatómicas donde el desarrollo de los folículos depende de la estimulación androgénica: Tercio proximal en cara interna de muslos, abdomen, pecho, parte baja de la espalda y cara entre otros. El grado y la severidad se evalúa de acuerdo a la escala de Ferriman-Galwey (3).

Virilización: La aparición de hipertrofia clitorídea, amenorrea prolongada, aumento de la musculatura, atrofia de los senos, hirsutismo severo y habitus masculino obliga a descartar la presencia de hiperplasia adrenal, hipertecosis o tumores ováricos o adrenales (3).

Irregularidad menstrual y anovulación: Las mujeres con SOP presentan grados variables de disfunción ovulatoria, manifestada como oligomenorrea, dismenorrea y amenorrea. Incluso, frecuentemente presentan infertilidad (17.5\% vs $1.3 \%$

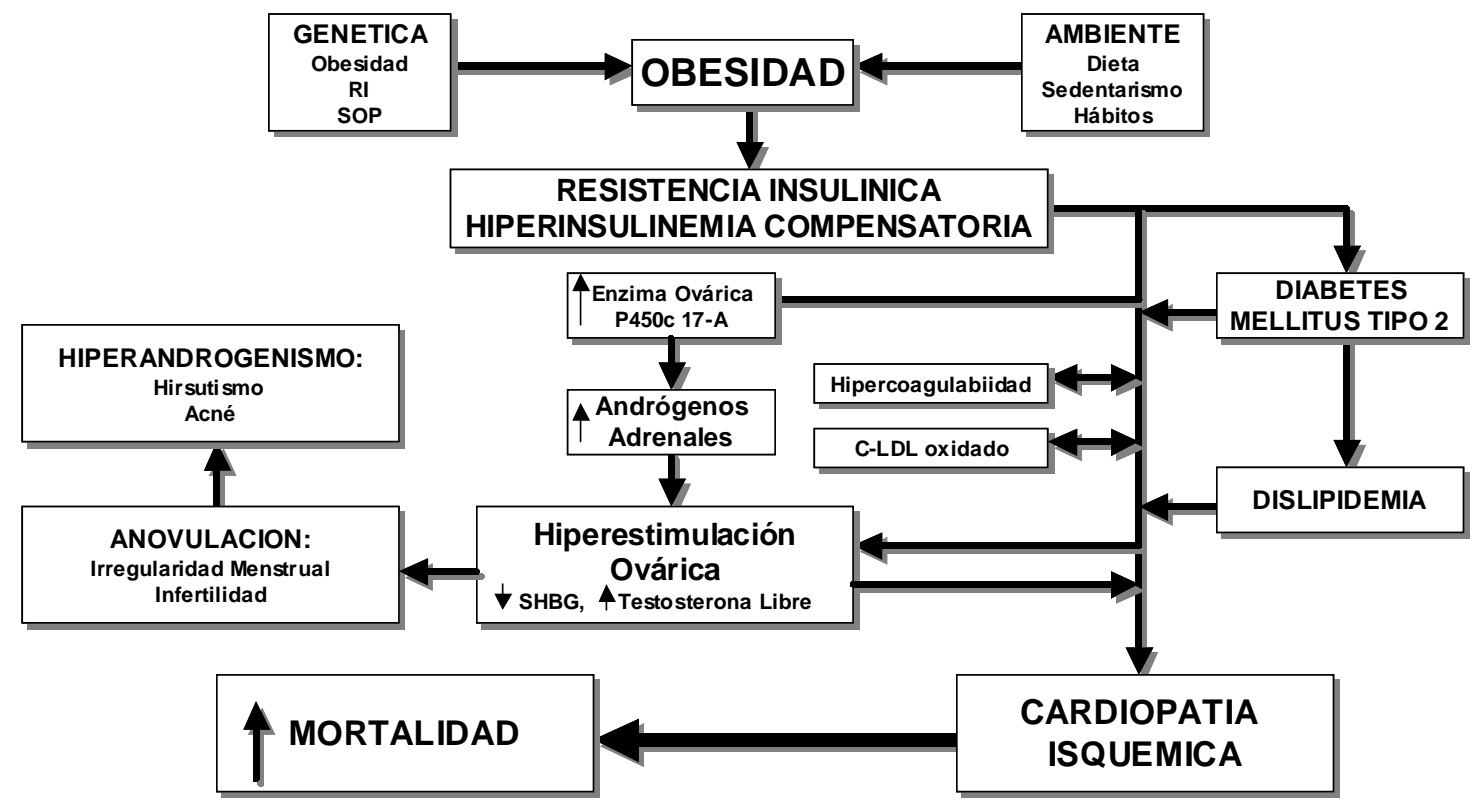

Figura 1.- Papel de la resistencia insulínica (RI) en la fisiopatología del Síndrome de Ovarios Poliquísticos (SOP) y el Síndrome Metabólico. Se esquematiza la determinante influencia de la RI y la hiperinsulinemia compensatoria en la génesis de la DMT2 (promoviendo la fatiga de la célula Beta), la dislipidemia y los riesgos de infarto agudo del miocardio. El SOP resulta de la hiperestimulación ovárica con participación de las adrenales lo que incrementa los riesgos de salud en estas pacientes.

\section{Revista Biomédica}




\section{Síndrome de ovarios poliquísticos.}

en normales) (11).

La relación entre el exceso de insulina y la anovulación se atribuye en primer lugar a la hiperandrogenemia y en segundo a la estimulación del inhibidor 1 del activador del plasminógeno (PAI-1). Este inhibidor no solo regula la eliminación de los depósitos de fibrina de los vasos sanguíneos sino que a nivel del ovario, inhibe a las colagenasas responsables de la ruptura folicular (39).

Acanthosis Nigricans: Se trata de una hiperplasia hiperpigmentada de la piel, la cual aparece predominantemente en el cuello y en pliegues cutáneos como axilas y codos (2). La importancia de su detección radica en que su presencia correlaciona significativamente con los estados de resistencia a la insulina e hiperinsulinemia compensatoria $(42,43)$. La presentan cerca del $40 \%$ de las adolescentes nativasamericanas, $13 \%$ de las africo-americanas, $6 \%$ de las hispanas y menos del 1\% de las caucásicas (44). Aunque la ausencia de acanthosis nigricans no descarta la existencia de resistencia a la insulina, el encontrarla identifica dentro de las diversas etnias a los subgrupos con mayor severidad del trastorno y que cursan con los niveles más elevados de insulina (45).

Dependiendo de las poblaciones estudiadas aparece ligada a obesidad hasta en el 74\% de los casos (46) constituyéndose como un factor de riesgo independiente para DM tipo $2(47,48)$. Aproximadamente el $30 \%$ de las pacientes con SOP lo presentan (43).

En nuestra práctica cotidiana al abordar un paciente con hiperandrogenismo y/o obesidad no debemos omitir la inspección de la región posterior del cuello, ya que de los pacientes que desarrollan acanthosis nigricans el 93-99\% la presentan en ese sitio (49) (ver figura 2).

\section{ESTUDIOS DE LABORATORIO.}

Al momento de enfrentar un caso de hiperandrogenismo nuestra obligación más importante es descartar la presencia de algunas patologías, que si bien no son frecuentes, requieren de abordajes particulares y tratamientos específicos. En este grupo incluimos a los tumores benignos y malignos de las adrenales y el ovario, así como los casos de hiperplasia suprarrenal congénita en su variedad no clásica, entre otras.

El SOP es un diagnóstico de exclusión y no es necesaria la presencia de alteraciones en los niveles hormonales o quistes en los ovarios para considerar su presencia; basta integrar el síndrome durante la historia clínica y hacer con base en un protocolo de escrutinio un buen diagnóstico diferencial.

Escrutinio: Determinación en ayunas y durante los primeros 7 días del ciclo de:

a) LH y FSH en pool (el promedio de 3 muestras recolectadas con 20 minutos de diferencia) y Prolactina.

b) Testosterona total y libre.

c) Dehidroepiandrosterona sulfato (DHEAS) y 17 alfahidroxiprogesterona (17-HPO) (3).

En el 66\% de los pacientes se documenta una relación LH:FSH ( $\geq 2.5: 1)$, la cual es característica de SOP (2). La prolactina debe ser determinada en la evaluación de cualquier paciente con amenorrea; muchas de las causas de hiperandrogenismo pudieran incrementarla pero valores $\geq 100$ sugieren patología hipofisiaria.

Los niveles discretamente elevados de testosterona libre pueden ser interpretados como hiperandrogenemia y en pacientes obesas sugerir la disminución de SHBG por la resistencia a la insulina $(39,50)$. La testosterona total es la prueba más

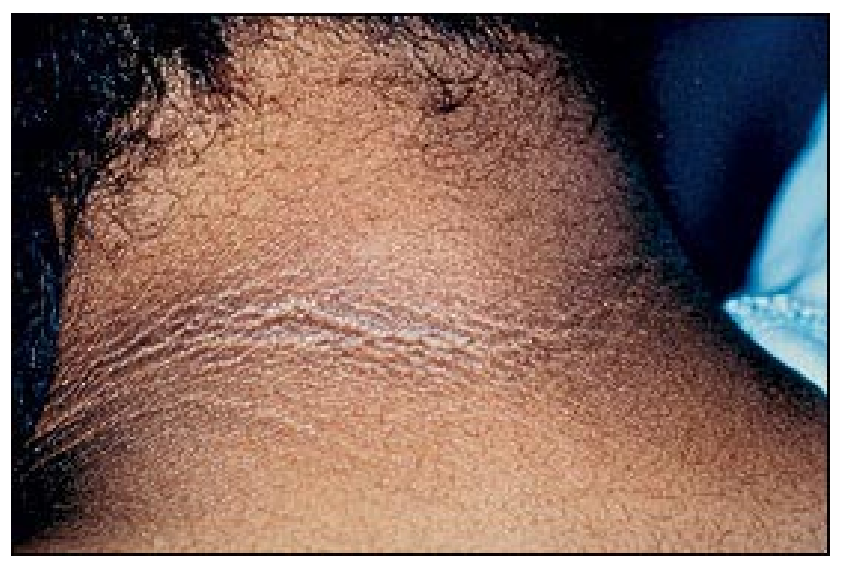

Figura 2.- Región posterior del cuello en una paciente con Síndrome de Ovarios Poliquísticos (SOP). Se aprecia el engrosamiento y la hiperpigmentación queratósica de la piel característica de la Acanthosis Nigricans.

Vol.14/No.3/Julio-Septiembre, 2003 


\section{MA Vargas-Carrillo, G Sánchez-Buenfil, J Herrera-Polanco, L Vargas-Ancona.}

validada para evaluar la presencia de tumores secretores de andrógenos: niveles $\geq 150 \mathrm{ng} / \mathrm{dL}$ (5.2 $\mathrm{nmol} / \mathrm{L}$ ) fuertemente sugieren la posibilidad de tumores ováricos o adrenales. La gran mayoría de las pacientes con SOP se encuentran por debajo de estas cifras (37)

Los niveles elevados de DHEAS indican la participación de las adrenales y en los casos en que las cifras sean desproporcionadamente altas $(\geq 800$ $\mathrm{mcg} / \mathrm{dL}$ ) debe descartarse la presencia de una neoplasia adrenal (37).

La 17-HPO con valores arriba de lo normal pero por debajo de $200 \mathrm{ng} / \mathrm{dL}$ prácticamente descartan la posibilidad de hiperplasia adrenal congénita por deficiencia de 21 hidroxilasa en su variedad no clásica; resultados de $1,000 \mathrm{ng} / \mathrm{dL}$ o superiores la confirman. Todos los pacientes con resultados intermedios se consideran dudosos y requieren de la realización de una prueba de estimulación con 250 mcgs de ACTH sintética (Cortrosyn), la cual amplifica el defecto enzimático $(3,51)$. En la figura 3 se esquematiza los puntos de corte en los niveles hormonales para considerar los principales diagnósticos diferenciales en pacientes con hiperandrogenismo. La presencia de los valores señalados debe alertar a la realización de pruebas específicas por el elevado riesgo. Sin embargo, su ausencia no descarta forzosamente estas etiologías por lo que los valores anormales en rangos intermedios deberán ser evaluados en forma individual.

Cuando la paciente desea procrear es fundamental determinar si los ciclos son ovulatorios. Los niveles de progesterona $<2 \mathrm{ng} / \mathrm{mL}$ después del día 21 del ciclo son interpretados como anovulación (3).

Estudios de Imagen: La ultrasonografía (USG) pélvica es el método de elección para la evaluación inicial de los anexos. Característicamente se trata de folículos de $8 \mathrm{~mm}$ con aumento del estroma central, aunque con la técnica vaginal pueden detectarse quistes de 3-5 mm. Los quistes ováricos aparecen irregularmente en las pacientes con SOP encontrándose diferencias significativas de acuerdo a las series estudiadas (25-80\%) (37).

Es importante señalar que la mayoría de los tumores suprarrenales son menores de 1-2 cms, de tal suerte que el USG no es útil en la gran mayoría de los casos y no debe utilizarse con este fin. El estudio de imagen de elección para el estudio de las suprarrenales es la tomografía axial computarizada de alta resolución. No debe indicarse si no se ha documentado en primera instancia la presencia de anormalidades en los tests endocrinos para evitar el riesgo de encontrarnos con incidentalomas, ya que hasta el $4.36 \%$ de la población pudiera presentarlos sin que esto represente un riesgo para su salud. La presencia de un tumor adrenal mayor de $3 \mathrm{~cm}$ sugiere malignidad $(52,53)$.

\section{MEDIDAS TERAPÉUTICAS EN EL SINDROME DE OVARIOS POLIQUÍSTICOS.}

Pérdida de peso. La obesidad es prevalente en mujeres con SOP, y se asocia con un empeoramiento de los síntomas. Mujeres con un índice de masa corporal (IMC) igual o mayor de $27 \mathrm{~kg} / \mathrm{m}^{2}$ son consideradas con obesidad de acuerdo al Consenso Mexicano (27). La pérdida de peso de un 5-7\% con respecto al basal produce un descenso en la concentración circulante de andrógenos, insulina y lípidos, lo cual se acompaña de una mejoría de la sintomatología y de las posibilidades de presentar ciclos ovulatorios (2). Las dietas altas en proteínas pudieran tener beneficios adicionales en cuanto a los parámetros endocrinos (54).

Tratamiento hormonal. Los anticonceptivos hormonales combinados proveen varios beneficios en las pacientes con SOP y durante mucho tiempo han sido la piedra angular del tratamiento. Restituyen los ciclos menstruales con eficienciay mejoran el hirsutismoen más del $60 \%$ de los casos por su efecto inhibidor de la LH, lo que aumenta los niveles de SHBG (38). También suprimen el metabolismo de los andrógenos en las adrenales y disminuyen el número de receptores de la 5a-reductasa en la piel, lo que beneficia al acné (3).

El tratamiento debe iniciarse con dosis bajas que se incrementarán de acuerdo a las necesidades. Deben evitarse las formulaciones que contienen norgestrel y levonorgestrel por su actividad androgénica (37). En general, las progestinas protegen al endometrio oponiéndose a los efectos proliferativos de los

\section{Revista Biomédica}


Síndrome de ovarios poliquísticos.

estrógenos pero tienen un efecto negativo en los parámetros metabólicos ya que aumentan la resistencia a la insulina y los niveles de triglicéridos $(55,56)$.

Agentes sensibilizadores de la insulina. Está documentado que la disminución en los niveles de insulina mediante el uso de este grupo de fármacos se acompaña de una mejoría del cuadro clínico y de las anormalidades metabólicas. Particularmente, en mujeres obesas con SOP han demostrado ser efectivos y pudieran ser considerados dentro de las opciones de elección para el inicio del manejo (57, 59).

Metformín. Es un agente anti-hiperglucemiante de la familia de las biguanidas. Actúa principalmente en el hígado inhibiendo parcialmente la gluconeogénesis, lo que reduce en un $17-25 \%$ la producción de glucosa y en el músculo esquelético donde incrementa la captación de glucosa estimulada por insulina en un $29 \%$ y disminuye la oxidación de los ácidos grasos en un 10-20\%. Globalmente esta cadena de eventos provoca en los pacientes diabéticos una mejoría en los niveles de glucosa plasmática en ayuno a la vez que mantiene las concentraciones de insulina sin cambio o incluso con una tendencia a la baja como consecuencia de la mejoría en su accionar (60).

En mujeres con SOP el metformín ha demostrado inducir la ovulación y mejorar las posibilidades de lograr un embarazo, incluso en pacientes sometidas a fertilización in vitro (61-63). En un estudio realizado en 23 mujeres con SOP tratadas con 1.5 grs de metformín por 6 meses, se demostró mejoría en las irregularidades menstruales en el $50 \%$ de los casos, mejoría significativa de la sensibilidad a la insulina medida por pinzamiento euglucémico, aumento de la SHBG y reducción de la testosterona libre (37).

En 10 pacientes con ciclos menstruales normales posterior al tratamiento con metformin se documentó que el $80 \%$ de ellas tenía ciclos ovulatorios. Los factores que pronosticaron la respuesta al metformín en este estudio fueron la hiperinsulinemia, las concentraciones menores de androstenediona y una menor severidad en las irregularidades menstruales (64). No hubo modificaciones en cuanto al hirsutismo en este estudio, pero en otros sí se ha documentado una disminución de la androgenemia, insulinemia y perfil lipídico, lo cual se traduce en una mejoría del hirsutismo $(65,66)$.

La combinación de metformín con anticonceptivos orales o acetato de ciproterona (67, 68) se ha acompañado de una mejoría en los parámetros clínicos y metabólicos. En pacientes con SOP y deseo de procrear, el agregar metformin a la terapia con clomifeno incrementó la posibilidad de ovulación en una muestra de 46 pacientes (90\% vs

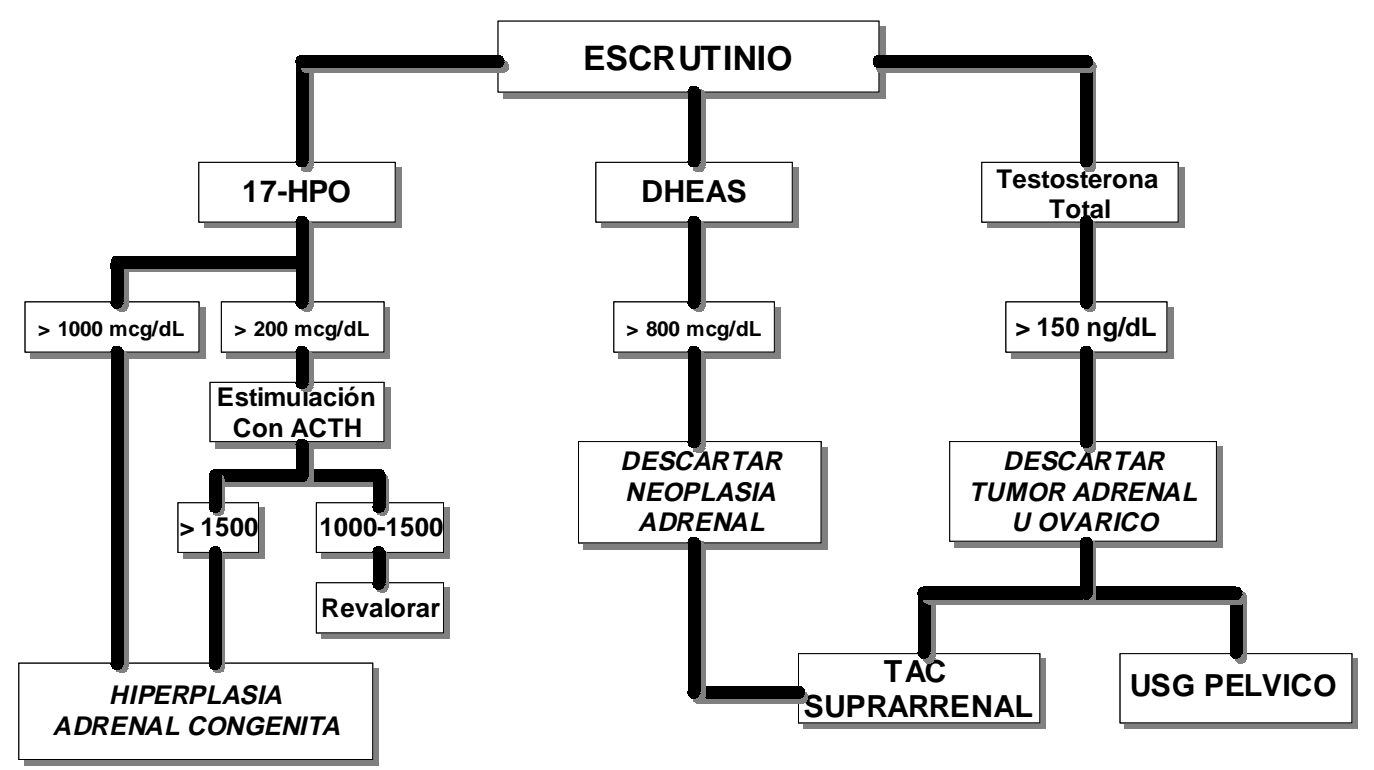

Figura 3.- Diagnóstico diferencial en hiperandrogenismo.

Vol.14/No.3/Julio-Septiembre, 2003 


\section{MA Vargas-Carrillo, G Sánchez-Buenfil, J Herrera-Polanco, L Vargas-Ancona.}

8\% en el grupo de clomifeno + placebo) (69).

La mejoría en la resistencia a la insulina inducida por el metformín también tiene efectos benéficos en el comportamiento del eje hipotálamo-hipófisis-adrenal $(70,71)$.

Tiazolidinedionas. Pertenecen a un nuevo grupo de fármacos anti-diabéticos derivados de la ciglitazona, particularmente útiles en estados de resistencia a la insulina por sus efectos en el metabolismo hepático de la glucosa y su acción en tejidos periféricos. Ejercen sus acciones principalmente en los tejidos periféricos (músculo esquelético) y en segundo término a nivel hepático activando a receptores nucleares específicos de la familia Gama-PPAR (Peroxime proliferator activator receptors), lo que inicia la trancripción genética de proteínas glucotransportadoras (GLUT-1 y GLUT4), regulando la homeostasia de los lípidos y la diferenciación de los adipocitos, lo que lleva un mejor funcionamiento de la insulina (72).

En pacientes con SOP la troglitazona, el primer medicamento del grupo y el más estudiado (actualmente no disponible para su venta en México) mejora la resistencia a la insulina disminuyendo la dehidroepiandrosterona sulfato, la testosterona libre, la androstenediona y la $\mathrm{LH}$, e incrementando la proteína transportadora de hormonas sexuales (73). Estos efectos disminuyen el hiperandrogenismo mejorando la anovulación y el hirsutismo (74) e induciendo una mejoría en la función endotelial (75). La administración de rosiglitazona a pacientes con SOP también se ha acompañado de mejoría en el perfil bioquímico, alivio de hiperandogenismo y de la anovulación $(76,77)$.

Tratamiento de la anovulación. El citrato de clomifeno, solo o en combinación con la pérdida de peso, sigue siendo, dada su seguridad y simplicidad, el tratamiento de primera elección en la infertilidad de origen anovulatorio asociado al SOP.

Actúa predominantemente como un antiestrógeno uniéndose a los receptores hipotalámicos, lo cual disminuye el mecanismo de retroalimentación negativa ejercida por los estrógenos endógenos. Esto aumenta la secreción de GnRH, la que a su vez estimula la secreción de LH y FSH (37).

El tratamiento con citrato de clomifeno comienza durante la fase proliferativa precoz (del 2do al 5to día del ciclo menstrual) con la administración de $50 \mathrm{mg} /$ día durante 5 días. Si la anovulación persiste, la dosis puede ser incrementada de 50 en $50 \mathrm{mg}$ al día hasta llegar a una dosis máxima de $250 \mathrm{mg} /$ día y al contrario que las gonadotrofinas exógenas, el aumento en la dosis de clomifeno no suele asociarse con un aumento del reclutamiento folicular. Como la finalidad del tratamiento es la inducción de la ovulación y el embarazo, la falta de respuesta terapéutica a pesar de las dosis máximas hace necesaria la adición de otros fármacos (78).

La administración de glucocorticoides a bajas dosis puede ser beneficiosa en las mujeres con anovulación hiperandrogénica (79). El mecanismo de acción implica la supresión de la síntesis de andrógenos suprarrenales, lo cual provoca una reducción de hasta el $40 \%$ del total de andrógenos circulantes $(79,80)$. Igualmente las dosis bajas de glucocorticoides pueden aumentar la síntesis y secreción de FSH (79). Generalmente se inicia el tratamiento con dexametasona a dosis de 0.25 a 0.5 $\mathrm{mg}$ o prednisona $5 \mathrm{mg}$ durante la noche junto con una progestina para inducir la menstruación antes de restituir el citrato de clomifeno (81).

Ocasionalmente, es necesaria la aplicación, de acuerdo a los niveles preovulatorios de estradiol y a la observación sonográfica de un folículo preovulatorio de una dosis ovulatoria de hCG (5.000 - 10.000 UI). La adición de la hCG puede ser de verdadera utilidad en caso de que no existiera un pico de $\mathrm{LH}$ (82).

La GnRH pulsátil ha sido utilizada para inducción de la ovulación en mujeres con SOP con tasas aproximadas de ovulación de hasta un 50\%. Estas tasas son de alguna manera más bajas que aquellas alcanzadas con el citrato de clomifeno porque la $\mathrm{GnRH}$ exógena no puede eliminar el trastorno en la secreción de gonadotropinas (83). La asociación de GnRH y clomifeno se relaciona con una tasa de éxito de hasta el $66 \%(82-84)$.

\section{Revista Biomédica}




\section{Síndrome de ovarios poliquísticos.}

\section{TRATAMIENTO DEL HIRSUTISMO.}

El hirsutismo se presenta en más del $70 \%$ de las mujeres con SOP. En una proporción de éstas, se acompaña por otros trastornos de la piel como alopecia, seborrea y acné, siendo todos ellos el resultado de una producción elevada de andrógenos y/o un aumento en la sensibilidad de la piel a los andrógenos. Hay varios antiandrógenos que reducen el hirsutismo. Los anticonceptivos orales incrementan los niveles de SHBG con un resultante descenso de los niveles de andrógenos libres. También el progestágeno que contienen los hormonales combinados inhibe a la 5a-reductasa en piel lo que mejora el hirsutismo y el acné (3).

Acetato de ciproterona. Es un derivado de la 17hidroxiprogesterona que tiene acciones progestágenas, antigonadotrópicas y antiandrogénicas. La mejoría clínica se debe a la inhibición de la testosterona y de la unión de la dihidrotestosterona (DHT) a su receptor intracelular; la concentración reducida de los andrógenos circulantes es el resultado de la inhibición de los andrógenos ováricos a través de la supresión de la liberación de LH (85).

Este fármaco es lipofílico y tiene un efecto progestágeno muy prolongado, por lo que debe ser interrumpido esporádicamente para asegurar la menstruación. La dosis moderadas $(50 \mathrm{mg} /$ día durante 10 días) de acetato de ciproterona acompañadas de un anticonceptivo oral suprimen la LH y testosterona tanto como los análogos de la GnRH. Esta terapia de combinación reduce los niveles plasmáticos de testosterona y androstendiona, suprime las gonadotropinas y aumenta la SHBG. El ACP utilizado como monoterapia a menudo ocasiona amenorrea particularmente en mujeres obesas. Con dosis más elevadas ( $100 \mathrm{mg} / \mathrm{día}$ ) se alcanza marcada mejoría, incluso en el hirsutismo severo $(86,87)$.

Los efectos colaterales incluyen ganancia de peso, pérdida de la libido, cefalea, náusea y fatiga, y se asocian al tratamiento con dosis máximas. Sin embargo, cuando se añade etinil-estradiol estas molestias prácticamente desaparecen. Debido a su naturaleza lipofílica, es necesario suspender el tratamiento por lo menos tres meses antes de intentar el embarazo. En la práctica se recomienda continuar el tratamiento con hormonales combinados al finalizar una terapia con ACP. Se sugiere el monitoreo de la función hepática antes de iniciar el tratamiento y a los dos, tres y posteriormente cada seis meses, dado el riesgo de hepatotoxicidad asociado a las dosis más elevadas (87).

Espironolactona. Es un diurético ahorrador de potasio, antagonista de la aldosterona y con potente acción antiandrogénica. Actúa compitiendo con los receptores androgénicos en su sitio de acción. La dosis recomendada es de 100-200 mg/día dividida en 2 dosis (88).

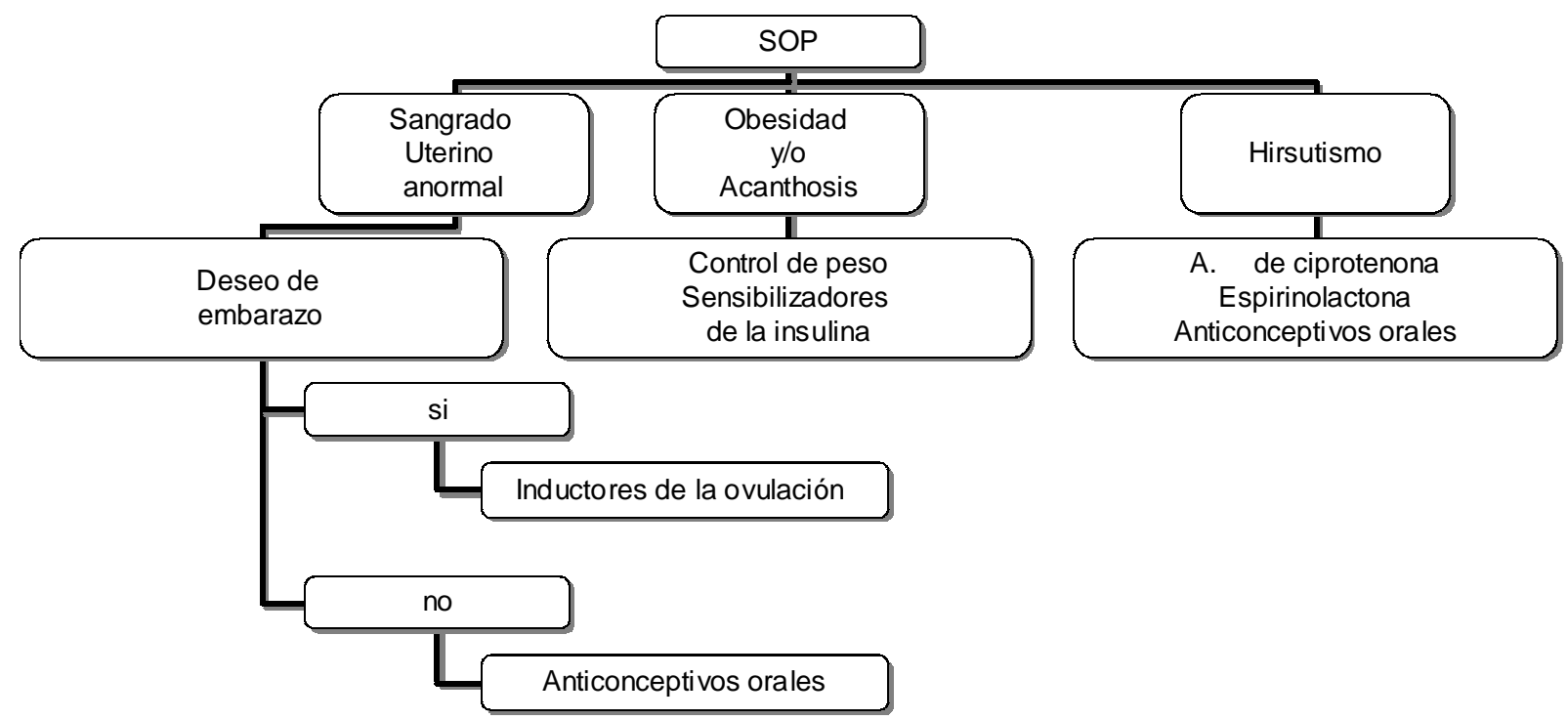

Figura 4.- Medidas terapeúticas en el Síndrome de Ovarios Poliquísticos (SOP). 


\section{MA Vargas-Carrillo, G Sánchez-Buenfil, J Herrera-Polanco, L Vargas-Ancona.}

Entre sus efectos adversos destaca la hiperkalemia, por lo que es necesario el monitoreo de los electrolitos séricos con su uso prolongado (89).

Agonistas de la GnRh. Los agonistas de la GnRH como el leuprolide, disminuyen la producción ovárica de esteroides por supresión de LH y FSH. Este tratamiento es altamente efectivo en mujeres con SOP severo o hipertecosis ovárica. La supresión de la producción de esteroides ováricos se asocia a sintomatología posmenopáusica como bochornos y resequedad vaginal.

Tratamiento Quirúrgico. La resección en cuña bilateral de los ovarios es un procedimiento quirúrgico que se realizó con éxito en pacientes con SOP durante muchos años. Esto provoca una reducción en los niveles de LH y producción de andrógenos, sin embargo por las múltiples complicaciones y adherencias relacionadas con el procedimiento ha caído en desuso. Actualmente en casos seleccionados se recurre a la cauterización de los folículos con electrocoagulación (37).

En la figura 4 se enfatizan las opciones terapéuticas en pacientes con SOP de acuerdo a los signos clínicos predominantes.

\section{CONCLUSIONES.}

El síndrome de ovarios poliquísticos es una entidad frecuente en nuestro medio y a la luz de los conocimientos recientes debemos considerar la participación de la resistencia a la insulina y la hiperinsulinemia compensatoria al momento de la toma de decisiones en el consultorio. Es un problema multidisciplinario que involucra a diversos trabajadores de la salud y que conlleva la responsabilidad de la prevención, por lo que debe siempre hacerse hincapié en la modificación de la dieta, la promoción del ejercicio, el monitoreo de la presión arterial, la determinación de la glucosa postprandial de dos horas (normal $\leq 140 \mathrm{mg} / \mathrm{dL}$ ) como escrutinio para DM tipo 2 y la realización de un perfil de lípidos con la finalidad de intervenir tempranamente.

\section{REFERENCIAS.}

1.- Dewailly D. Definition of polycystic ovary syndrome. Hum Fertl 2000; 3: 73-6.
2.- Balen A, Rajkowha M. Polycystic ovary syndrome-a systemic disorder? Best Pract Res Clin Obstet Gynaecol 2003; 17:263-74.

3.- American Association of Clinical Endocrinologists Medical guidelines for clinical practice for the diagnosis and treatment of hyperandrogenic disorders. Hyperandrogenic Disorders Task Force. Endocrine Practice 2001; 7: 120-34.

4.- Wild RA. Obesity, lipids, cardiovascular rik and androgen excess. Am J Med. 1995; 98: 27S-32S.

5.- Consenso Mexicano de Resistencia a la Insulina y Síndrome Metabólico. Rev Mex Cardiol 1999; 10: 3-19.

6.- Slowey MJ. Polycystic Ovary syndrome: new perspective on and old problem. South Med J 2001; 94: 190-6.

7.- Gambineri A, Pelusi C, Vicennati V, Pagotto U, Pasquali R. Obesity and the polycystic ovary syndrome. Int J Obes Relat Metab Disord 2002; 26: 883-96.

8.- Ehrmann DA, Barnes RB, Rosenfield RL. Prevalence of impaired glucose tolerance and diabetes in women with polycystic ovary syndrome. Diabetes Care 1999 22: 141-6. 9.- Legro RS, Dunaif A. The role of insulin resistance in polycystic ovary syndrome. Endocrinologist 1996; 6: 307-21. 10.- Wild RA. Metabolic and cardiovascular issues in women with androgen excess. Endocrinologist. 1996; 6: 86-92.

11.- Wild S, Perpoint T, Jacobs H, McKeigue P. Long term consequences of polycystic ovary syndrome: results of a 31 year follow-up study. Hum Fertil 2000; 3: 101-5.

12.- Dahlgreen E, Janson PO, Lapidus L, Oden A. Polycystic ovary syndrome and risk for myocardian infarction. Acta Obstet Gynecol Scand 1992; 71: 599-603.

13.- Birdsall MA, Farquhar C, White H. Association between polycystic ovaries and extent of coronary artery disease in women having cardiac catheterization. Ann Intern Med 1997; 126: $32-5$.

14.- Harvad Health Letter. In Brief: Irregular periods linked to Heart Attacks. Harvard Health Online Books and Newsletters. November 2002. p. 874-8.

15.- Legro RS, Kunselman AR, Dunaif A. Prevalence and predictors of dyslipidemia in women with polycystic ovary syndrome. Am J Med. 2001; 111: 665-6.

16.- Dejaguer S, Pichard C, Giral P, Bruckert E, Federspield MC, Beucler I, et al. Smaller LDL particle size in women with polycystic ovary syndrome compared to controls. Clin Endocrinol 2001; 54: 455-62.

17.- Sabuncu T, Vural H, Harma M, Harma M. Oxidative stress in polycystic ovary syndrome and its contribution to the risk of cardiovascular disease. Clin Biochem 2001; 34: 407-13.

18.- Paradisi G, Steinberg HO, Hempfling A. Polycistic ovary syndrome is associated with endothelial dysfunction. Circulation 2001; 103: 1410-5.

19.- Taskinen MR. Triglyceride is the major atherogenic lipid in NIDDM. Diabetes/Metabolism Reviews, 1997; 13: 93-8.

20.- Betteridge D. Cholesterol is the major atherogenic lipid in NIDDM. Diabetes/Metabolism Reviews, 1997; 13: 99-104. 


\section{Síndrome de ovarios poliquísticos.}

21.- Ambrosch A, Muhlen I, Kope D. LDL size distribution in relation to insulin sensitivity and lipoprotein pattern in young and healthy subjects. Diabetes Care 1998; 21: 2077-84.

22.- Vrbikova J, Bendlova B, Hill M, Vankova M, Vondra K, Starka L. Insulin sensitivity and beta cell function in women with polycystic ovary syndrome. Diabetes Care 2002; 25: 1217-22.

23.- Kelly CJ, Lyall H, Petrie JR, Gould GW, Connell JM, Rumley A, et al. A specific elevation in tissue plasminogen activator antigen in women with polycistic ovary syndrome. J Clin Endocrinol Metab 2002; 87: 2387-90.

24.- Loverro G, Lorusso F, Mei L, Depalo R, Cormio G, Selvaggi $\mathrm{L}$. The plasma homocysteine levels are increased in polycystic ovary syndrome. Gynecol Obstet Invest 2002; 53: 157-62.

25.- Kashyap S, Claman P. Polycistic ovary disease and the risk of pregnancy induced hypertension. J Reprod Med 2000; 45: 991-4.

26.- Cibula D, Skrha J, Hill M, Fanta M, Haakova L, VrbIkova $\mathrm{J}$, Zivny J. Prediction of insulin sensitivity in nonobese women with polycystic ovary syndrome. J Clin Endocrinol Metab 2002; 87: 5821-5.

27.- Quibrera R, Vargas L, Avila H. Epidemiología de la obesidad en México. En Vargas L, Bastarrachea R, Laviada $\mathrm{H}$, Gonzalez J, editores Obesidad Consenso. México: Mc Graw Hill; 2002. p. 25-48.

28.- Vargas-Ancona L. Epidemiología de la Diabetes Mellitus, Intolerancia a la glucosa y factores de riesgo aterogénico en Yucatán, México. Rev Biomed 1994: 5: 151-9.

29.- Laviada H, Vargas L, Arroyo P, Pardío J, Fernández V. Prevalencia de Diabetes Mellitus Tipo 2 en la población urbana de Yucatán. Revista de Endocrinología y Nutrición 1999; 7: 82

30.- Valles S, Vargas L, Laviada H, Pardío J. Prevalencia de Dislipidemias en la población urbana de Yucatán. Revista de Endocrinología y Nutrición 1999; 7: 82.

31.- Poretsky L, Piper B. Insulin resistance, hypersecretion of LH, and a dual-defect hypothesis for the pathogenesis of polycystic ovary syndrome. Obstet Gynecol 1994; 84:61321

32.- Poretsky L. Insulin resistance and hyperandrogenism: Update 1994. Endoc Rev 1994; 2: 26-35

33.- Poretsky L, Kalin MF. The gonadotropic function of insulin. Endoc Rev 1987; 8: 145-9

34.- Barbieri RL, Makris A, Randall RW, Daniels G, Kistner RW, Kenneth JR. Insulin stimulates androgen accumulation in incubations of ovarian stroma obtained from women with hyperandrogenism. J Clin Endocrinol Metab 1986; 62: 904-9. 35.- Poretsky L, Grigorescu F, Seibel M, Moses AC, Flier JS. Distribution and characterization of insulin and insulin-like growth 1 receptors in normal human ovary. J Clin Endocrinol Metab. 1985; 61:355-62

36.- Rosenbaum D, Haber RS, Dunaif A. Insulin resistance in polycystic ovary syndrome: decreased expresión of Glut-4 glucose transporters in adipocytes. Am J Physiol 1993; 264: 197-202.

37.- Taylor AE. Polycystic ovary syndrome. Endoc Metab Clin N A 1998; 27: 877-902.

38.- Poretsky L. The insulin-related ovarian regulatory system in health and disease. Endoc Rev 1999; 20: 535-82.

39.- Moran C, Asís R. The role of the adrenal cortex in polycistic ovary síndrome. Obstet Gynecol Clin N A 2001; 28: 63-75.

40.- McCarney CR, Eagleson CA, Marshall JC. Regulation of gonadotropin secretion: implications for polycystic ovary syndrome. Sem Reprod Med 2002; 20: 317-26.

41.- Shaw JC. Acne: effect of hormones on pathogenesis and management. Am J Clin Dermatol 2002; 3: 571-8.

42.- Katz AS, Goff DC, Feidman SR. Acanthosis nigricans in obese patients: presentations and implications for prevention of atherosclerotic vascular disease. Dermatol Online J 2000; 6: $25-8$

43.- Rendon MI, Cruz PD Jr, Sonheimer RD, Bergstresser PR. Acanthosis Nigricans: A cutaneous marker of tissue resistance to insulin. J Am Acad Dermatol 1989; 21: 461-9.

44.- Stuart CA, Driscoll MS, Lundsquit KF, gilkison CR, Shaheb S, Smith MM. Acanthosis nigricans. J Basic Clin Physiol Pharmacol 1998; 9: 407-18.

45.- Varthakavi PK, Patel KL, Wadhwa SL, Shopkar U, Sengupta RA, Merchant PC, Mahtalia SD, Nihalani KD. A study of insulin resistance in subjects with acanthosis nigricans. J Assoc Physicians India 2001; 49: 705-12.

46.- Hud JA Jr, Cohen JB. Prevalence and significance of acanthosis nigricans in an adult obese population. Arch Dermatol 1992; 128: 941-4.

47.- Gilkison C, Stuart CA. Assessment of patients with acanthosis nigricans skin lesions for hiperinsulinemia, insulin resistance and diabetes risk. Nurse Pract 1992; 17: 26-8.

48.- Dunaif A, Graf M, Mandeli J. Characterization of groups of hyperandrogenic women with acanthosis nigricans, impaired glucose tolerance, and/or hyperinsulinemia. J Clin Endocrinol Metab 1987; 65: 499-507.

49.- Burke JP, Hale D. A quantitative scale of acanthosis nigricans. Diabetes Care, 1999; 22: 1655-9.

50.- Penttila TL, Koskinen P. Obesity regulates bioavailable testosterone levels in women with oy without polycystic ovary syndrome. Fertil Steril 1999; 71: 457-61.

51.- Asís R. 21-Hidroxylase-deficient nonclassic adrenal hyperplasia. Endocrinologist 1995; 5:297-303.

52.- Kloss R, Gross M. Incidentally discovered adrenal masses. Endoc Rev 1995; 18: 460-81.

53.- Barzon L, Scaroni C. Risk factors and long-term follow up of adrenal incidentalomas. J Clin Endocrinol Metab 1999; 84: 520-6.

54.- Moran LJ, Noakes M, Clifton PM. Dietary composition in restoring reproductive and metabolic physiology in overweight women with polycystic ovary syndrome. J Clin 


\section{MA Vargas-Carrillo, G Sánchez-Buenfil, J Herrera-Polanco, L Vargas-Ancona.}

Endocrinol Metab 2003; 88: 812-9.

55.- Bagis T, Gockcel A. The effects of short term medroxyprogesterone acetate and micronized progesterone on glucose metabolism and lipid profiles in patients with polycystic ovary syndrome: A prospective randomized study. J Clin Endocrinol Metabolism 2002; 87: 4536-40.

56.- Mastorakos G, Koliopoulos C, Creatsas G. Androgen and lipid profiles in adolescents with polycystic ovary syndrome who were treated with two forms of combined oral contraceptives. Fertil Steril 2002; 77: 919-27.

57.- Bloomgarden ZT, Futterweit W. Use of insulin-sensitizing agents in patients with polycystic ovary syndrome. Endoc Pract 2001; 7: 279-86.

58.- Ciotta L, De Leo V. Endocrine and metabolic effects of insulin sensitizers in the treatment of patients with polycystic ovary syndrome and hyperinsulinemia. Gynecol Obstet Invest 2001; 51: 44-50.

59.- Nestler JE, Stovall D. Strategies for the use on insulin sensitizing drugs to treat infertility in women with polycystic ovary syndrome. Fertil Steril 2002; 77: 209-15.

60.- Blonde L, Guthrie RD, Sandberg MI. Metformin: an effective and safe agent for initial monotherapy in patients with non-insulin-dependent diabetes mellitus. Endocrinologist 1996; 6: 431-8.

61.- Ibáñez L, Valls C, Ferrer A. Sensitizacion to insulin induces ovulation in nonobese adolescents with anovulatory hyperandrogenism. J Clin Endocrinol Metab 2001; 86: 35958 .

62.- Batunkan C, Baysal B. Metformin improves ovulation and pregnancy rates in patients with polycystic ovary syndrome. Arch Gynecol Obstet 2001; 265: 124-7.

63.- Stadtmauer LA, Tona SK, Riehl RM, Tarbert LM. Impact of metformin therapy on ovarian stimulation and outcome in coasted patients with polycystic ovary syndrome undergoing in vitro fertilization. Reprod Biomed Online 2002; 5: 112-5.

64.- Moghetti P, Castello R, Negri C. Metformin effects on clinical features, endocrine and metabolic profiles and insulin sensitivity in polycystic ovary syndrome: A randomized, double-blind, placebo-controlled 6 month trial, followed by open, long term clinical evaluation. J Clin Endocrinol Metab 2000; 85: 139-46.

65.- Loverro G, Lorusso F, De Pergola G, Nicolardi V, Mei L, Selvaggi L. Clinical and endocrinological effects of 6 months of metformin treatment in young hyperinsulinemic patients affected by polycystic ovary syndrome. Gynecol Endocrinol 2002; 16: 217-24.

66.- Kelly CJ, Gordon D. The effect of metformin on hirsutism in polycystic ovary syndrome. Eur J Endocrinol 2002; 147: 217-21.

67.- Elter K. Clinical, endocrine and metabolic effects of metformin added to ethinyl estradiol-cyproterone acetate in non-obese women with polycystic ovary syndrome: A randomized controlled study. Hum Reprod 2002; 17: 1729-37.
68.- Ibañez L, Valls C. Additive effects of insulin sensitizing and anti androgen treatment in young, nonobese women with hyperinsulinism, hyperandrogenism, dylipidemia and anovulation. J Clin Endocrinol Metab 2002; 87: 2870-4.

69.- Nestler JE, Jacubowicz DJ, Evans WS, Pasquali R. Effects of Metformin on spontaneous and clomiphene-induced ovulation in the polycystic ovary syndrome. N Eng J Med 1998; 338: 1876-80.

70.- Vrbikova J, Hill M,Starka I. The effects of long term metformin treatment on adrenal and ovarian steroidogenesis in women with polycystic ovary syndrome. Eur J Endocrinol 2001; 144: 619-28.

71.- Arslanian SA. Metformin therapy in obese adolescents with polycystic ovary syndrome and impaired glucose tolerance: amelioration of exaggerated adrenal response to ACTH with reduction of insulinemia/insulin resistance. J Clin Endocrinol \& Metab. 2002; 87: 1555-9.

72.- Park KS, Ciaraldi TP, Lindgren K. Troglitazone effects on gene expression in human skeletal muscle of type 2 diabetes involve up regulation of peroxime proliferator activated receptor gamma. J Clin Endocrinol Metab 1998; 83: 2830-5. 73.- Dunaif A, Scott D, Finegood D. The insulin-sensitizing agent troglitazone improves metabolic and reproductive abnormalities in the polycystic ovary syndrome. J Clin Endoc Metab 1996; 81: 3299-306.

74.- Azziz R, Ehrmann D. Troglitazone improves ovulation and hirsutism in the polycystic ovary syndrome: A multicenter, double blind, placebo controlled trial. J Clin Endocrinol Metab 2001; 86: 1626-31.

75.- Paradisi G, Steinberg HO. Troglitazone therapy improves endothelial function to near normal levels in women with polycystic ovary syndrome. J Clin Endocrinol \& Metab. 2003; 88: 576-80.

76.- Conway GS. Polycystic ovary syndrome: Clinical aspects. Clin Endocrinol Metabol 1996; 10:263-79.

77.- Moghetti P, Castello R, Negri C. Metformin effects on clinical features, endocrine and metabolic profiles and insulin sensitivity in polycystic ovary syndrome: A randomized, double-blind, placebo-controlled 6 month trial, followed by open, long term clinical evaluation. J Clin Endocrinol Metab 2000; 85: 139-46.

78.- Bateman BG, Nunley WCJ, Kolp LA. Exogenous estrogen therapy for treatment of clomiphene citrate-induced cervical mucus abnormalities: is ti effective? Fertil Steril 1990; 54:55780.

79.- Polson DW, Kiddy DS, Mason HD, Franks S. Induction of ovulation with clomiphene citrate in women with polycystic ovary syndrome: the difference between responders and nonresponders. Fertil Steril 1999; 51: 30-4.

80.- Sing KB, Dunnihoo DR, Mahajan DK, Bairnsfather LE. Clomiphene-Dexamathasone treatment of clomiphene resistant women with polycystic ovary syndrome. J Reprod Med 1992; 37:215-8. 
81.- Issacs JD, Lincolns SR, Cowan BD. Extended clomiphene citrate and prednisona for treatment of chronic anovulation resistant to clomiphene citrate alone. Fertil Steril 1997; 67: 641-3.

82.- Agarwal SK, Buyalos RP. Corpus luteum function and pregnancy rates with clomiphene citrate teraphy: comparison of human chorinic gonadotropin-induced versus spontaneous ovultation. Hum Reprod 1995; 10: 111-30.

83.- Homburg R, Esthel A, Armar NA, Tucker M. One hundred pregnancies after treatment with pulsatile luteinizing hormone releasing hormone to induce ovulation. Br Med J 1999; 289: 809-12.

84.- Tan SL, Farhi J, Homburg R, Jacobs HS, Induction of ovulation in clomiphene resistant polycystic ovary syndrome with pulsatile GnRH. Obstet Gynecol 1996; 88: 221-6

85.- Prevelic GM, Puzigaca Z, Balint-Peric LJA. Effects of an oral contraceptive containing cyproterone acetate on the symptoms, hormone profile and ovarian volumen of hirsute women with polycystic ovarian syndrome. Ann NY Acad Sci 1997; 687: 255-62.

86.- Wiebe RH, Morris CV. Effect of anoral contraceptive on adrenal and ovarian androgenic steroids. Obstet Gynecol 1994; 63:12-4.

87.- Jeffcoate W. Treatment of women with hirsutism. Clin Endocrinol 1993; 38:727-35.

88.- Lobo RA, Shoupe D, Serafini P, Brinton D, Horton R. Effects of spironolactone on serum androgens and anagen hair in hirsute women. Fertil Steril 1999; 43:200-5.

89.- Shaw JC. Spironolactone in dermatologic therapy. J Am Acad Dermatol 2001; 24:236-43. 